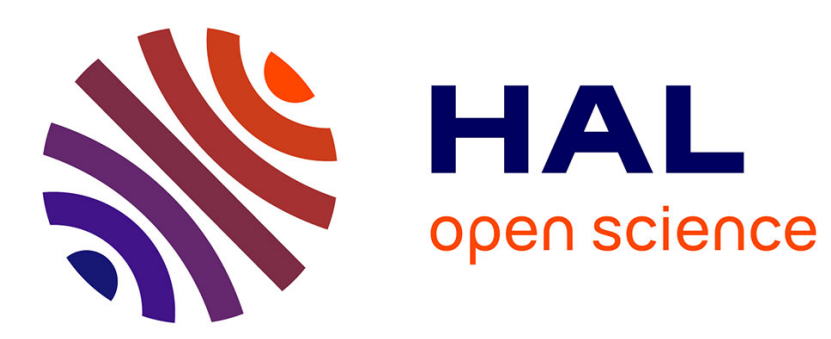

\title{
A new scintillating fiber dosimeter using a single optical fiber and a CCD camera
}

\author{
A.M. Frelin-Labalme, J.M. Fontbonne, G. Ban, A. Batalla, J. Colin, A. \\ Isambert, M. Labalme, T. Leroux, A. Vela
}

\section{- To cite this version:}

A.M. Frelin-Labalme, J.M. Fontbonne, G. Ban, A. Batalla, J. Colin, et al.. A new scintillating fiber dosimeter using a single optical fiber and a CCD camera. IEEE Transactions on Nuclear Science, 2006, 53, pp.1113-1117. 10.1109/TNS.2006.874931 . in2p3-00089774

\section{HAL Id: in2p3-00089774 https://hal.in2p3.fr/in2p3-00089774}

Submitted on 23 Aug 2006

HAL is a multi-disciplinary open access archive for the deposit and dissemination of scientific research documents, whether they are published or not. The documents may come from teaching and research institutions in France or abroad, or from public or private research centers.
L'archive ouverte pluridisciplinaire HAL, est destinée au dépôt et à la diffusion de documents scientifiques de niveau recherche, publiés ou non, émanant des établissements d'enseignement et de recherche français ou étrangers, des laboratoires publics ou privés. 


\title{
A New Scintillating Fiber Dosimeter Using a Single Optical Fiber and a CCD Camera
}

\author{
A.-M. Frelin, J.-M. Fontbonne, G. Ban, A. Batalla, J. Colin, A. Isambert, M. Labalme, T. Leroux, and A. Vela
}

\begin{abstract}
Radiotherapy treatments become more and more accurate, using very small irradiation fields and complex dose depositions. So small dosimeters for real time and in vivo dosimetry, suitable for photons as well as for electrons beams are highly desired. In this context, a scintillating fiber dosimeter (SFD) has been developed by the Laboratoire de Physique Corpusculaire de Caen (LPC Caen), France, in collaboration with one of the French regional center for cancer treatment Centre Régional de lutte contre le cancer F. Baclesse (CRLCC F. Baclesse), Caen, France, and the ELDIM Company, Hérouville, France. This plastic dosimeter is water equivalent, and it is suitable for photons as well as for electrons beams without correction. It is a real time dosimeter, with an excellent signal to noise ratio, and a spatial resolution of about a few millimeters. The aim of this study was to reduce the size of the scintillator in order to improve the spatial resolution of this dosimeter. So, a new light collection device has been developed to reduce the length of the scintillator from $1 \mathrm{~cm}$ to $1 \mathrm{~mm}$ without loss in the signal to noise ratio. The accuracy of this improved prototype has been tested by comparison with standard ionization chambers and the difference between the two devices never exceeded one percent for photon and for electron irradiation beams. A first set of commercial SFD is under completion at ELDIM and it will be soon clinically tested in several French centers for cancer treatment.
\end{abstract}

Index Terms-CCD camera, Cerenkov cancellation, dosimetry, scintillating fiber, .

\section{INTRODUCTION}

A SCINTILLATING fiber dosimeter (SFD) has been devetoped by the Laboratoire de Physique Corpusculaire de Caen (LPC Caen), France, in collaboration with the French center for cancer treatment Baclesse (Centre Régional de Lutte Contre le Cancer F. Baclesse, (CRLCC F. Baclesse), Caen, France, and the ELDIM Company, Hérouville, France. This dosimeter uses a small (few $\mathrm{mm}^{3}$ ) plastic scintillator which emits a light amount proportional to the absorbed dose. This light is guided by an optical fiber, and then measured by a photodetector. Because of its size, its water equivalence, and its neutral properties, this dosimeter is a very good alternative to ionization chambers which clearly cannot be used for in vivo control or high spatial resolution dosimetry because of the high voltage needed and their size.

The first prototype developed by the LPC was build around a $1 \mathrm{~cm}$ long, $1 \mathrm{~mm}$ diameter scintillating fiber and a photodiode

Manuscript received October 30, 2004.

A.-M. Frelin, J.-M. Fontbonne, G. Ban, J. Colin, and M. Labalme are with the Laboratoire de Physique Corpusculaire de Caen, 14050 Caen Cedex, France (e-mail: fretin@lpccaen.in2p3.fr).

A. Batalia, A. Isambert, and A. Vela are with the Centre de Lutte Contre le Cancer, 14076 Caen Cedex 05, France.

T. Leroux is with the ELDIM Society, Hérouville 14200, France.

Digital Object Identifier 10.1109/TNS.2006.874931 with integrated spectral filters [1]. Expressed in terms of radiological units, its dose rate noise was about $170 \mu \mathrm{Gy} / \mathrm{s}^{-1}$ (i.e., $10 \mathrm{mGy} / \mathrm{min}^{-1}$ ), and its spatial resolution was of the order of a few millimeters. This resolution was better than ionization chamber's, but new treatments using small irtadiation fields require a spatial resolution as high as possible. The only way to obtain this spatial resolution is to decrease the scintillator's volume. But this reduction induces a proportional diminution of the scintillation amount. The photodetector has been redesigned to manage this constraint, and the photodiode has been replaced by a CCD detector.

This contribution deals with this new light collection device, and its performances, for photons and electrons irradiation beams.

\section{DEVICE DESCRIPTION}

The SFD, represented in Fig. 1, consists of three parts: the sensor (A), the optical line (B), and the signal processing part (C).

The whole system has been designed to maximize the SNR and to cope with the limitations of each part.

\section{A. The Sensor}

It is a $1 \mathrm{~mm}$ long and $1 \mathrm{~mm}$ diameter scintillating fiber (Bicron BCF-60) glued to $2 \mathrm{~m}$ long clear optical fiber.

This scintillator has been chosen because its emission spectrum (from 500 to $600 \mathrm{~nm}$ ) is sufficiently separated from the (1) $/\left(\lambda^{2}\right)$ spectrum of the Cerenkov radiation and from the maximum attenuation of the optical fiber (at about $600 \mathrm{~nm}$ ) [2].

At radiotherapy energies the amount of scintillation light is energy independent and proportional to the dose absorbed by the scintillator. The main difficulty to use this information is that under irradiation the scintillator and the clear optical fiber also emit Cerenkov light for electrons above $180 \mathrm{keV} \mathrm{[3].} \mathrm{This}$ parasitic contribution is unavoidable, and it must be known or at least subtracted from the scintillation light if one wants to measure dose from the scintillation. The optical device and a specific calibration method have been developed for this purpose, and are described later.

\section{B. The Optical Line}

This is an essential part because it deports the expensive part of the device (the CCD camera) from the irradiation room. Unfortunately the length of the line is a drawback because it induces an attenuation of the light transmitted, and consequently a decrease of the signal to noise $(S / N)$ ratio. 


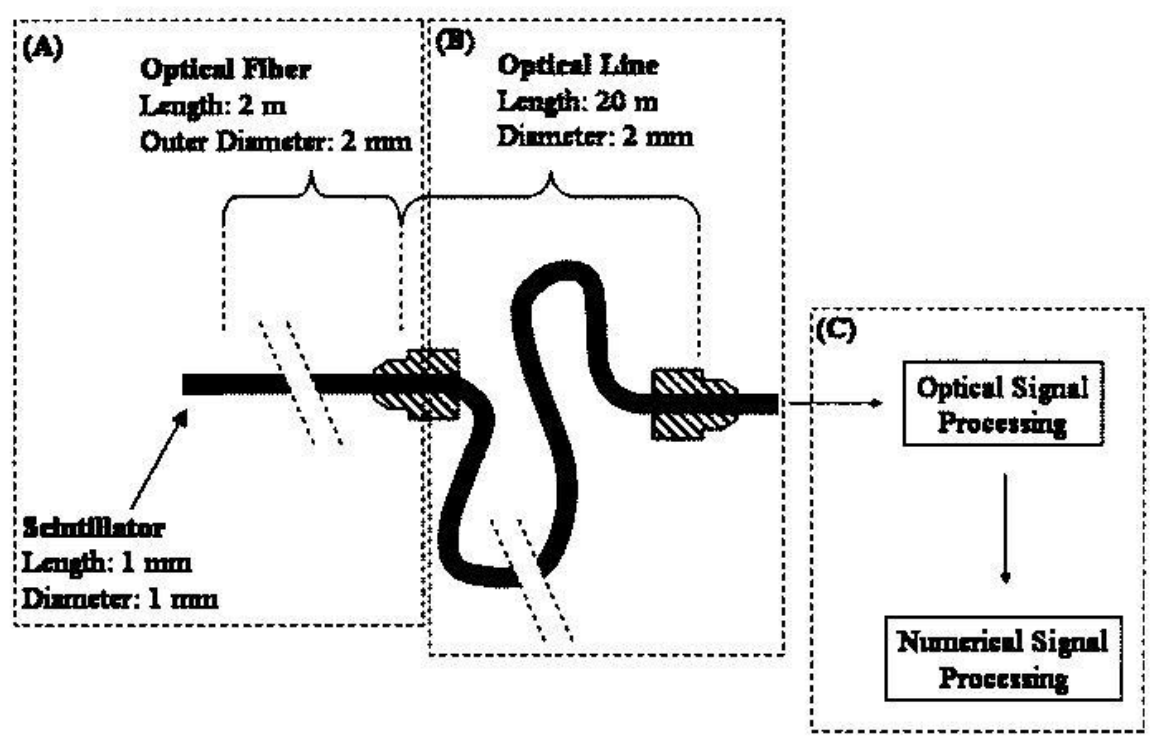

Fig. 1. Schematic of the SFD.

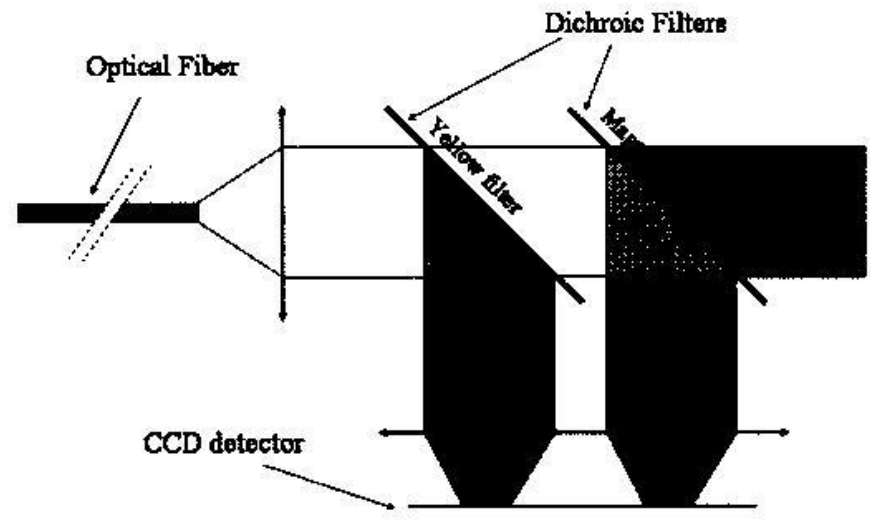

Fig. 2. The new optical signal processing device. A yellow dichroic filter reflects wavelengths below $500 \mathrm{~nm}$ toward the CCD detectors and transmits the wavelengths above $500 \mathrm{~nm}$. A magenta dichroic filter reflects the wavelengths from 500 to $600 \mathrm{~nm}$ loward the CCD detextor and transmits the wavelengths above $600 \mathrm{~nm}$.

The line is a commercially available optical fiber: $1 \mathrm{~mm}$ diameter core with $0.1 \mathrm{~mm}$ clear cladding and $2 \mathrm{~mm}$ black outer covering. Its attenuation depends on the wavelength of the transmitted light. It has a $0.1 \mathrm{~dB} / \mathrm{m}^{-1}$ minimum attenuation around $500 \mathrm{~nm}$ (the wavelength domain of the scintillation).

\section{The Signal Processing Part}

The signal processing device, represented in Fig. 2, is the most original part of this dosimeter. It is divided into an optical processing part and a numerical processing part.

The optical processing part separates the light transmitted by the optical fiber into two wavelength domains $(\lambda<500 \mathrm{~nm}$ and $500<\lambda<600 \mathrm{~nm}$ ) by means of two Edmund Industrial Optics subtractive dichroic filters. The corresponding "blue" and "green" light amounts ( $B$ and $G$ ) are accurately measured with the CCD detector, and finally the amount of Cerenkov light is subtracted and the dose calculated with a new analysis method.
The CCD detector is a $576 \times 768$ pix $\mathrm{E} 2 \mathrm{~V}$ product $\mathrm{L} 3 \mathrm{Vision}$ Type. It has a $16 \mathrm{MHz}$ maximum readout rate, a readout noise $<\mathrm{le}^{-}$(at maximum readout rate). Its quantum efficiency $(\mathrm{QE})$ is illustrated by Fig. 3

The numerical processing part subtracts the Čerenkov light contribution from $B$ and $G$, and calculates the dose $D$ in radiological units.

$D$ is related to $B$ and $G$ by the relation

$$
D=\alpha \cdot B+\beta \cdot G
$$

where $\alpha$ and $\beta$ are the calibration coefficients.

The calibration [1] consists of measuring $D$ with an ionization chamber and the $B$ and $G$ light amounts for two different irradiation fields providing two different proportions of Čerenkov light relatively to $D$ :

$$
\left(\begin{array}{l}
D 1 \\
D 2
\end{array}\right)=\left(\begin{array}{ll}
B 1 & G 1 \\
B 2 & G 2
\end{array}\right) \cdot\left(\begin{array}{l}
\alpha \\
\beta
\end{array}\right)
$$

The calibration procedure allows the computation of $\alpha$ and $\beta$, and then one can on line calculate $D$.

\section{LIGHT AMOUNT AND SIGNAL MEASURED}

The quality of the dose measurement depends on the spectral separation of the scintillation and the Cerenkov. But it depends also on the amount of each. The larger these quantities, the higher the global S/N ratio. So it is essential to evaluate them.

\section{A. Light Produced}

On one hand, it is assumed that about $100 \mathrm{eV}$ are necessary to produce one photon of scintillation in plastic scintillators [4]. As the stopping power for electrons in this medium is about 


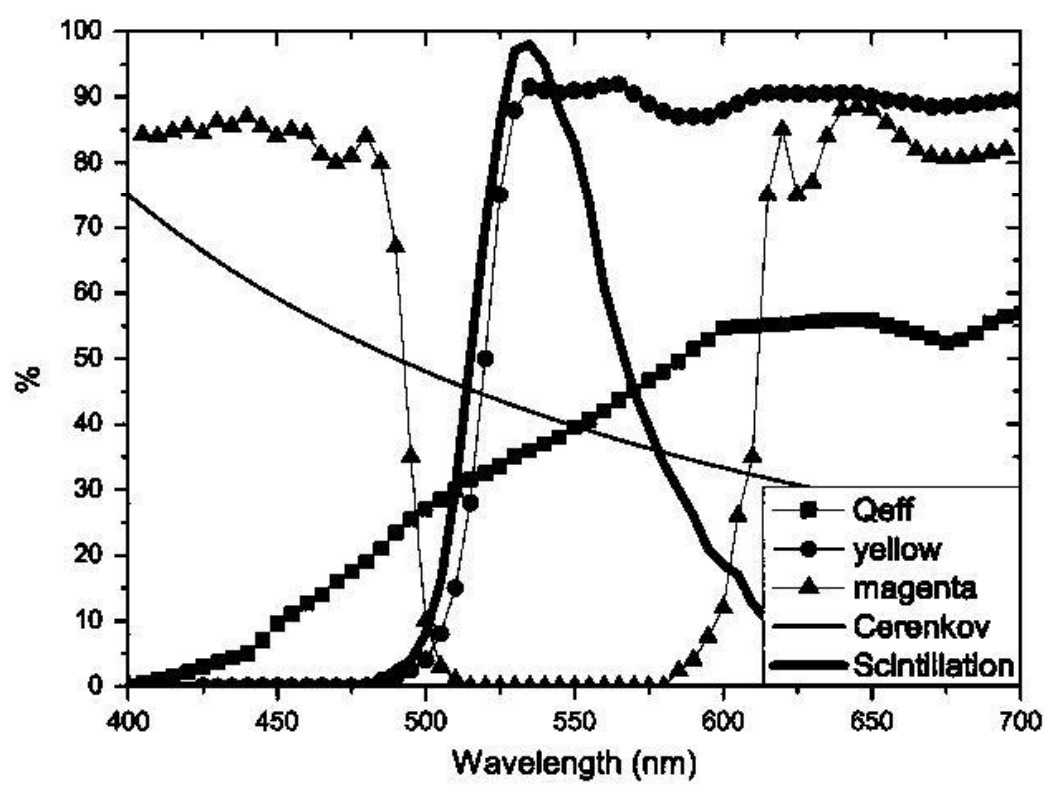

Fig. 3. Spectral sensibility of the CCD detector, transmission spectra of the yellow and magenta dichroic filters, and scintillation spectrum.

$2 \mathrm{MeV} \cdot \mathrm{cm}^{2} / \mathrm{g}$ at radiotherapy energies, ${ }^{1}$ a single electron produces about 2000 scintillation photons per millimeter path.

On the other hand, the amount of Črenkov light (number of photons $N$ created per unit path length $x$ and per unit wavelength $\lambda$ ) is given by the relation [5]:

$$
\frac{\partial^{2} N}{\partial x \cdot \partial \lambda}=2 \pi \alpha\left(1-\frac{1}{\beta^{2} n^{2}}\right) \frac{1}{\lambda^{2}}
$$

with $\alpha$ being the fine structure constant, $\beta$ the ratio of the particle velocity to the speed of light in vacuum, and $n$ medium index of refraction.

- The integration of this relation shows that a single relativistic electron produces 27 Cerenkov photons, from 400 to $700 \mathrm{~nm}$, per path millimeter.

Furthermore, a 1 Gy dose deposition in our $7.910^{-4} \mathrm{~cm}^{3}$ scintillator will produce about $4.910^{10}$ photons of scintillation, and about $6.6210^{8}$ Čerenkov photons for each irradiated fiber millimeter. (That means that $74 \mathrm{~mm}$ of irradiated fiber generate as much light as the scintillator does.)

\section{B. Light Measured}

Radiotherapy accelerators have a typical $2 \mathrm{~Gy} / \mathrm{mn}^{-1}$ doserate. According to the previous paragraph, a clinical irradiation generates $1.62 \times 10^{9}$ "green" photons $/ \mathrm{s}^{-1}$ (from 500 to $600 \mathrm{~nm}$ ) of scintillation, and the same quantity of "blue" photons (below $500 \mathrm{~nm}$ ) in $74 \mathrm{~mm}$ of irradiated fiber (which is not an atypical length), due to the Čerenkov effect. However the totality of this light yield is not guide to the CCD: Roughly one half of the produced light is guided in the right direction, i.e., toward the photo detector.

All the optical components attenuate the signal. A very optimistic evaluation provides the following values:

- There are two connectors and a plastic-air interface. It is equivalent to five plastic-air or air-plastic interfaces. For

INIST databases; available: http:/www.nist.gov/srd/physics.htsn a single attenuation of $4 \%$ the total interfaces transmission is $80 \%$.

- In the most favorable wavelength domain, around $500 \mathrm{~nm}$, the optical fiber exhibits losses of $0.1 \mathrm{~dB} / \mathrm{m}^{-1}$. So a $20 \mathrm{~m}$ line transmits $75 \%$ of the signal.

- The fiber has an acceptance angle of $42^{\circ}$. Only light that reach the exit surface of the fiber at an angle that is equal to or less than the acceptance angle is transmitted to the air. It corresponds to only $10 \%$ of the light guided by the line.

- The light transmitted from the optical line to the air is emitted in a $2 \pi$ solid angle. The first lens $(2 \mathrm{~cm}$ diameter) is $2 \mathrm{~cm}$ far from the optical fiber, so the light has a probability of reaching the detector of about $15 \%$.

- On average the dichroic filters transmit $80 \%$ of the light in their transmission range and reflect almost $100 \%$ of the light in the orthogonal range.

- The CCD camera has a poor efficiency. It is $10 \%$ on average below $500 \mathrm{~nm}$, and $40 \%$ on average from 500 to 600 nm.

Consequently, the signal in the "blue" channel is about $6 \times 10^{5}$

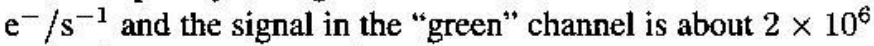
$\mathrm{e}^{-} / \mathrm{s}^{-1}$ for a $2 \mathrm{~Gy} / \mathrm{min}^{-1}$ dose rate.

Finally, the "blue" and "green" beams are received by 50 pixels (px) diameter surfaces of the CCD detector, which has a readout of about 30 acquisitions (Acq) per second. So one can consider $10 \mathrm{e}^{-} / \mathrm{Acq}^{-1} / \mathrm{px}^{-1}$ for the "blue" channel, and 40 $\mathrm{e}^{-} / \mathrm{Acq}^{-1} / \mathrm{px}^{-1}$ for the "green" channel.

\section{Signal to Noise Ratio}

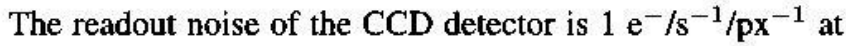
maximum readout rate. Conversely with the previous section, this quantity can be expressed in radiological units. It corresponds to $0.85 \mathrm{mGy} / \mathrm{s}^{-1}$. In fact, we have measured an a noise of $1 \mathrm{mGy} / \mathrm{s}^{-1}$.

The dose is not measured with only one image acquisition, but with $k$ acquisitions. Consequently, the relevant signal measured 

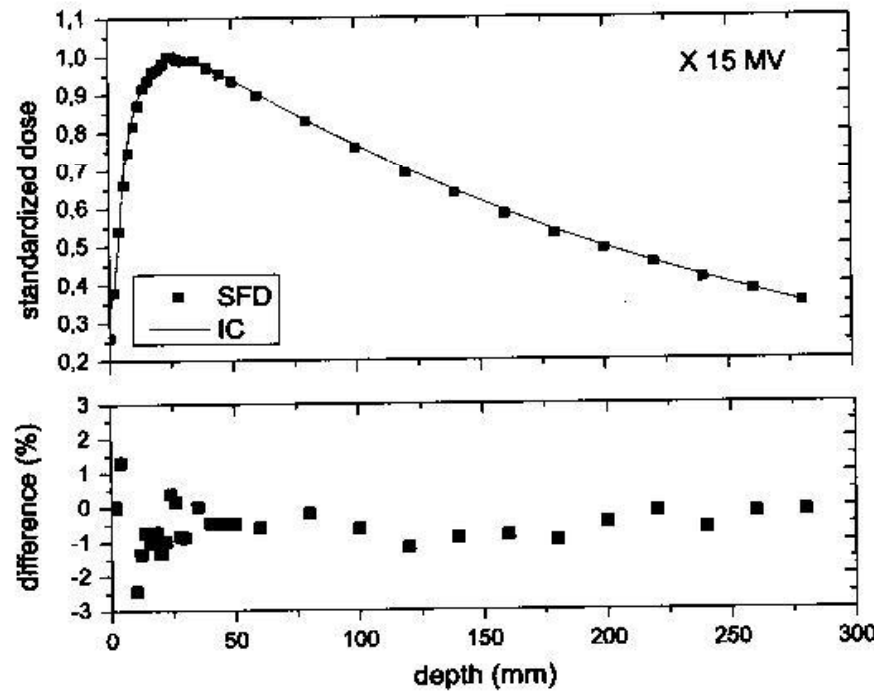

Fig. 4. Depth dose measurement comparison between SFD and a $0.125 \mathrm{~cm}^{3}$ PTW ionization chamber, and difference relative to the maximal dose with 15 MV photons.

is $S=k . s$, the readout noise is $N=k . n$, and the total signal measured is $S_{T}=k \cdot(s+n)$.

The uncertainty on this quantity is given by: $\sigma_{S_{T}}=$ $\sqrt{k \cdot(s+n)}$.

Finally, the signal to noise ratio is

$$
\frac{S}{\sigma_{S_{T}}}=\frac{k \cdot s}{\sqrt{k \cdot(s+n)}}=\frac{s}{\sqrt{s+n}} \cdot \sqrt{k}=\frac{1}{\varepsilon} .
$$

A single acquisition induces $\varepsilon=34 \%$ for the "blue" channel and $16 \%$ for the "green" channel, which is clearly insufficient. The 30 acquisitions (corresponding to about 1 s) induce $\varepsilon=6 \%$ for the "blue" and 3\% for the "green." And 300 acquisitions (corresponding to about 10s) is a good compromise between the measuring time and S/N ratio: $\varepsilon=2 \%$ for the "blue," and less than $1 \%$ for the "green."

\section{DEPTH DOSE MEASUREMENTS}

We have tested our SFD by comparing the standardized dose measured in a water phantom at different depths with an ionization chamber (a PTW $0.125 \mathrm{~cm}^{3}$ chamber when irradiated with photons and a PTW plane-parallel chamber with electrons) which is the reference dosimeter for radiotherapy.

The beam conditions were Beam X 8 MV, $15 \mathrm{MV}, 25 \mathrm{MV}$ and Electrons (E) $6 \mathrm{MeV}, 15 \mathrm{MeV}, 21 \mathrm{MeV}$, dose $0.4 \mathrm{~Gy}$ maximum; irradiation field $10 \mathrm{~cm} \times 10 \mathrm{~cm}$; phantom water surface DSP 100 .

We can see in the Figs. 4 and 5 that the differences between the two detectors are within $\pm 1 \%$ for photons and electrons beams of $15 \mathrm{MV}$ and $15 \mathrm{MeV}$ (except for the point at the water surface which is excluded from the statistics because neither the ionization chamber nor the SFD are completely in the water phantom). The differences between the two detectors for the other beams are summarized in the Table I which presents the mean and the standard deviation of the measured differences through all the depth. It is worth noting that the value given are
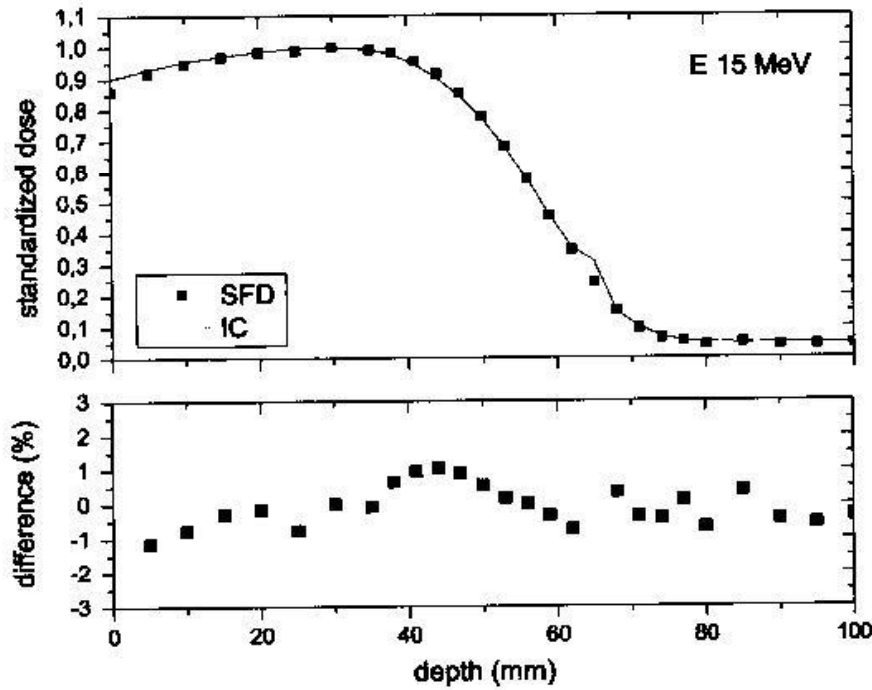

Fig. 5. Depth dose measurement comparison tetween SFD and a plane-paralle PTW jonization chamber, and difference relative to the maximal dose with 15 MeV electrons.

TABLE I

Mean and Standard Deviation of the Differences Between the Scintillating Fiber Dosimeter and the Ionization Chamber Through all the Depth

\begin{tabular}{ccc}
\hline & \multicolumn{2}{c}{ Difterences between SFD and IC } \\
\cline { 2 - 3 } & Merm & Stendard Deviation \\
\hline Beam X 8MV & $0,00 \%$ & $0,54 \%$ \\
Beam X 15 MV & $-0,08 \%$ & $0,85 \%$ \\
Bean X 25 MV & $0,00 \%$ & $0,68 \%$ \\
\hline Beam E 6 MeV & $0,00 \%$ & $0,73 \%$ \\
Beam E 15 MeV & $-0,27 \%$ & $0,62 \%$ \\
Beam E 21 MeV & $-0,19 \%$ & $0,81 \%$ \\
\hline
\end{tabular}

without any corrections for the SFD and only a calibration is necessary to measure the dose.

\section{CONCLUSION AND OUTLOOK}

The measurements have shown the accuracy of the SFD and its suitability for photons and electrons beams without corrections. It is flexible and robust, and its small size ( $1 \mathrm{~mm}$ length) promises an excellent spatial resolution, and it gives online dose monitoring in almost any treatment condition. All these properties make it a perfect detector for in vivo dosimetry.

Nevertheless with this configuration and to ensure a $1 \%$ dose accuracy, the dosimeter has a maximum readout rate of $0.1 \mathrm{~Hz}$. This rate is limited by the signal to noise ratio (especially by the one of the Blue signal as shown in the Section III-C), and can be easily improved by increasing signal to noise ratio.

We have measured a $1 \mathrm{mGy} / \mathrm{s}^{-1}$ noise for this device. This noise being almost entirely due to the readout noise of the CCD detector, it can be hardly reduced. So the only way to obtain a better SNR is to increase the signal amount and in particular the Blue amount. This can be performed with a CCD quantum efficiency matching the Čerenkov spectrum as well as the scintillation spectrum, and it will allow to decrease the integration time from 10 to $2 \mathrm{~s}$. Besides, it has been stated in the Section II-B that only $15 \%$ of the light is transmitted from the optical fiber 
to the first lens. This is very poor, and a well designed optical device will also reduce the signal loss and, consequently, the integration time of the dosimeter. At last, the transmission of the fiber junctions is not optimal and requires better connectors.

These improvements, essential to extent the use of our dosimeter to treatments like IMRT requiring higher readout rate, are under study, and an industrial prototype is being manufactured to be clinically tested at the beginning of 2005 .

Moreover, validation of the dosimeter for absolute dosimetry and a "3-D version" for 3-D measurements are under development.

\section{REFERENCES}

[1] J. M. Fontbonne, G. Iltis, G. Ban, A. Batalla, J. C. Vernhes, J. Tillier, N. Bellaize, C. Lebrun, B. Tamain, K. Mercier, and J. C. Motin, "Scintiliating fiber dosimeter for radiation therapy accelerator," IEEE Trans. Nucl. Sci., vol. 49, no. 5, pp. 2223-2227, Oct. 2002.

[2] S. F. de Boer, A. S. Beddar, and J. A. Rawlinson, "Optical filtering and spectral measurements of radiation-induced light in plastic scintil]ation dosimetry," Med. Phys., vol. 38, pp. 945-958, 1993.

[3] A. S. Beddar, T. R. Mackie, and F. H. Attix, "Cerenkov light generated in optical fibers and other light pipes irradiated by electron beams," Phys. Med. Biol, vol. 37, no. 4, pp. 925-935, 1992.

[4] K. Akimov, Scintillation Counters in High Energy Physics (in Ed). New York: Academic, 1964.

[5] D.E. Groom, "Review of particle physics," Eur. Physical J, vol. C15, no. 1,2000 . 